\title{
Adaptive TDMA Based QoS-Aware MAC protocol for Hierarchical Wireless Sensor Networks
}

\author{
Dilip Kumar ${ }^{1+}$ and Tarunpreet Kaur ${ }^{1}$ \\ ${ }^{1}$ SLIET Longowal, Punjab, India
}

\begin{abstract}
This paper presents an adaptive time division multiple access based energy efficient medium access control (ATDMA-MAC) protocol to handle the variable traffic load while maintaining the quality of service $(\mathrm{QoS})$ assurance for delay-sensitive applications. The ATDMA-MAC protocol dynamically allocates time slots to the sensor nodes based on their queue length information to achieve minimum latency for a wide range traffic load. Furthermore, the analytical model of energy consumption has derived to demonstrate the superiority of the ATDMA-MAC protocol. Simulation results show that the proposed ATDMA-MAC protocol sends more data against existing TDMA based MAC protocols with minimum transmission latency and energy consumption.
\end{abstract}

Keywords: wireless sensor networks, MAC, TDMA, latency, QoS

\section{Introduction}

In the past decade, wireless sensor networks (WSNs) have come across as one of the significant technologies which incorporate automatic sensing, processing, and wireless transmission into small electronic devices called sensor nodes [1]. The information received from the sensor nodes is transmitted to the base station for processing and analyzing [2]. The past research on WSN is mainly focussed towards the monitoring applications but due to the advancement in micro-electro-mechanical systems (MEMS), the wireless networking technologies enabled the widespread utilization of networks in different applications like healthcare, military surveillance, industrial automation, and smart cities [3]. However, in these delaysensitive applications energy efficiency, latency, and reliability are the primary constraints and to handle real-time data traffic in WSN, end-to-end latency within an acceptable range is required. Many researchers have tried to address these constraints by introducing different medium access control (MAC) protocols [4-5]

Generally, MAC protocols divide into two basic categories: contention based and scheduled based protocols. In contention-based protocols, each sensor node contends to access the channel. However, when several neighbouring nodes try to access the channel simultaneously, then there may be a chance of collision among the sensor nodes. This, not only cause a delay in data delivery but also depletes the sensor nodes energy very quickly [6]. The existing contention based MAC protocols are SMAC [7], TMAC [8], and BMAC [9]. The scheduled protocols avoid the probability of collisions by allocating a separate time slot to each sensor node. TDMA [10-11] is the most commonly used scheduled based MAC protocol which assigns guaranteed time slot (GTS) to access the medium for data transmission. GTS allocation provides collisionfree data transmission regardless of the dynamic traffic load, thereby guaranteeing high efficiency and low transmission delay. These observation motivate researchers to modify the TDMA based MAC protocols to enhance and provide QoS assurance for smart cities applications where loss or delay in data packets is intolerant. This paper presents an adaptive TDMA-based MAC (ATDMA-MAC) protocol to support variable data traffic in cluster-based WSNs. The key contributions of ATDMA-MAC are summarized below: (i) A novel TDMA frame structure comprises of control slots, announcement slot, and data transmission slots.

\footnotetext{
+ Corresponding author. Tel.: + 9417213652
}

E-mail address: dilip.k78@gmail.com. 
(ii) Assumes data slots of small size to handle the adaptive traffic load. (iii) The slots are dynamically allocated to sensor nodes based on their queue length information. (iv) Derives analytical model of energy consumption.

The paper is organized as follows: Related work is presented in Section II. Section III illustrates the detailed description of our new proposed ATDMA-MAC protocol. Section IV presents the energy consumption analytical model used in the ATDMA-MAC protocol. The Simulation results and analysis are discussed in Section V and Section VI concludes the paper.

\section{Related Work}

TDMA protocol is a typical scheduled based MAC protocol where fixed time slots are allocated to the sensor nodes in a cluster. However, unique time slot allocation avoids the collision, but energy consumption during idle period is not avoided as sensor node keeps its radio ON during its allocated slot even it does not have data to transmit. This constraint was addressed in Energy efficient TDMA (E-TDMA) [12]. In ETDMA, a member node keeps its radio OFF, when it has no data to transmit in the allocated time slot.

However, both E-TDMA and TDMA are unable to handle the variable traffic load. To overcome this issue, Bit-Map-Assisted (BMA) [13] and Bit-Map-Assisted with Round Robin (BMA-RR) [14] have been proposed. BMA utilizes unused slots of the sensor nodes by assigning these slots to more data requesting nodes. However, BMA protocol does not consider fairness during the slot allocation, which has been addressed in BMA-RR by allocating unused slots to more data requesting nodes by employing Round Robin technique. However, both of these protocols offer a fixed number of data slots in a round, which are unable to handle adaptive traffic load efficiently and thus, results in high transmission delay and low network throughput. Most of the existing TDMA based MAC protocols addresses energy consumption or unused time slots. They do not provide QoS assurance to real-time data traffic in WSN or cannot accommodate dynamic traffic load efficiently. Our proposed ATDMA-MAC protocol solves each of these existing shortcomings.

\section{Proposed ATDMA-MAC Protocol}

This section describes the proposed ATDMA-MAC protocol for the hierarchical communication network. The proposed protocol operation is divided into rounds, where each round consists of a setup phase (SP) and a steady-state phase (SSP) as illustrated in Fig.1.

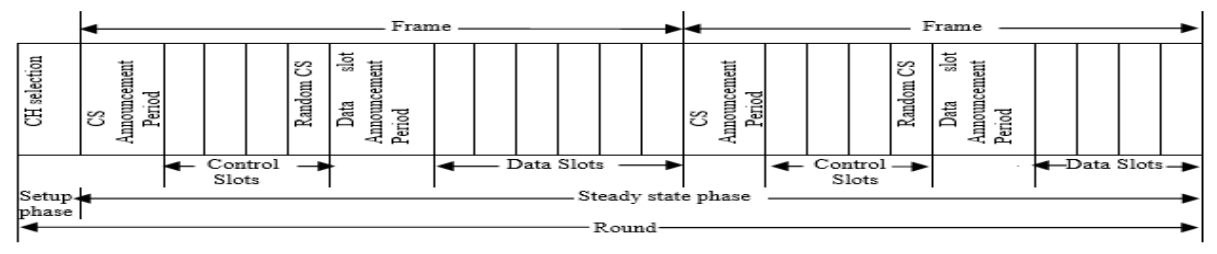

Fig. 1: Operation of ATDMA-MAC protocol.

During the setup phase, multiple clustering techniques have been proposed in WSNs for cluster head $(\mathrm{CH})$ election and cluster formation. In this paper, $\mathrm{CHs}$ are elected based on LEACH protocol [11]. After $\mathrm{CHs}$ election, each $\mathrm{CH}$ broadcasts a small advertisement message called $\mathrm{CH}$-Adv message in the network. the message comprises of ID of elected $\mathrm{CH}$ and its location information. Based on the received $\mathrm{CH}-\mathrm{Adv}$ messages from $\mathrm{CHs}$, the non-CH nodes send a Join_Req message contains its node ID to the nearest $\mathrm{CH}$. Further, the $\mathrm{CH}$ computes the number of associated member nodes by counting the Join_Req messages and then assigns a control time slot to each of its member nodes and also broadcasts this data to its member nodes through CS_Assign message which comprises of frame control, allocated control slot number, control slot duration (CSD), total number of control slots and frame check sequence (FCS). The length of CS_Assign message is based on the number of member nodes within the cluster. From the assignment message, the node also gets the information about the end of setup phase and initialization of steady-state phase as shown in Fig. 2.

The steady-state phase is the data transmission phase in ATDMA-MAC protocol where sensor nodes transmit the sensing data to their corresponding CHs. It consists of multiple frames for communication 
between the $\mathrm{CH}$ and its member nodes. Each frame consists of control period followed by an announcement period, and a data transmission period respectively. The control period is divided into fixed sized $\mathrm{M}$ control subslots, where $\mathrm{M}$ is the number of member nodes of $\mathrm{CH}$. These control slots are used to send Data_Req messages to their respective $\mathrm{CHs}$. The data request message contains queue length information of the sensor node. All data sending nodes are required to send request messages in their allocated control slots whereas, nodes with empty queue keep their radios off to save their energy.

However, during the entire control period, the $\mathrm{CH}$ remains in receiving mode in order to receive Data_Req messages from its member nodes. Based on the queue length information of the member nodes, each $\mathrm{CH}$ maintains a data sub-slots reservation list which includes the member node ID and the current numbers of packets in its queue. In ATDMA-MAC protocol, data slots are allocated to the source nodes on priority basis of their queue length. As a result, nodes with more number of packets in its queue are prioritized over nodes that have less number of packets in the queue. The reason to adopt queue length information for data slot assignment is that it avoids the packet loss due to queue overflow in case of variable traffic load. In order to minimize transmission latency, ATDMA-MAC protocol allows the sensor nodes to transmit their data as a whole instead of transmitting data in parts. However, if requesting data slots increases than the available slots, then $\mathrm{CH}$ considers these sensor nodes in the next session or in the next round.

The $\mathrm{CH}$ informs the source nodes about their allocated data slots by broadcasting a data slot allocation (DS_Alloc) message which comprises of node ID of the member node along with its starting data slot number. The DS_Alloc frame also informs the start time of its next control period to the member nodes which do not send data request to the $\mathrm{CH}$ in the current session as shown in Fig. 3.

By receiving DS_Alloc messages, the member node determines its allocated data slots and transmits data in that slots in order to avoid the contention. However, the proposed protocol keeps the data slot duration relatively small as compared to other TDMA protocols. The smaller sized data slots help in improving the network throughput by minimizing the unused time slot duration of the sensor node. As the $\mathrm{CH}$ keeps its radio $\mathrm{ON}$ to achieve the data packets from member nodes throughout the data slots. So smaller duration of data slots also consumes a significant amount of energy and consequently network lifetime is improved.

The $\mathrm{CH}$ aggregates the data received from the member nodes and transmits aggregated data to the BS by using a CSMA technique, as given in the LEACH protocol [13]. The next round begins after a predefined time and the same process is repeated for next rounds.

\section{Analytical Model of Energy Consumption}

During the setup phase, the total energy consumed by the $\mathrm{CH}\left(E_{c h}^{S P}\right)$ of $N$ size cluster is computed as:

$$
E_{c h}^{S P}=P_{c h}^{C A d v} \times T_{C A d v}+P_{c h}^{J R} \times T_{J R}(N-1)+P_{c h}^{C S} \times T_{C S}
$$

where, the parameters $P_{c h}^{C A d v}, P_{c h}^{J R}$, and $P_{c h}^{C S}$ indicate the power consumed by $\mathrm{CH}$ in broadcasting $\mathrm{CH} \_\mathrm{Adv}$ message, receiving Join_Req messages and transmitting CS_Alloc message to sensor nodes, respectively. The parameter $T_{C A d v}, T_{J R}$, and $T_{C S}$ indicate the time utilized to transmit CH_Adv message, receive Join_Req messages and transmit CS_Alloc message to sensor nodes, respectively.

Then, the energy consumed by member node $j,\left(E_{j}^{S P}\right)$ during the setup phase is defined by equation (2).

$$
E_{j}^{S P}=P_{j}^{C A d v} \times T_{C A d v}+P_{j}^{J R} \times T_{J R}+P_{j}^{C S} \times T_{C S}
$$

where, the parameters $P_{c h}^{C A d v}, P_{c h}^{J R}$, and $P_{c h}^{C S}$ indicate power consumed by member node $j$ in receiving $\mathrm{CH}$ Adv message, sending Join_Req message, and receiving CS_Alloc message, respectively. The parameters $T_{C A d v}, T_{J R}$, and $T_{C S}$ indicate the time utilized to receive $\mathrm{CH}_{-}$Adv message, transmit Join_Req messages and receive CS_Alloc message, respectively.

The total energy consumed in the setup phase, i.e. $E^{\text {Setup }}$, is given by equation (3).

$$
E^{\text {Setup }}=E_{c h}^{S P}+\sum_{j=1}^{N-1} E_{j}^{S P}
$$

A steady-state phase consists of multiple sessions for communication between sensor nodes and CHs. The energy consumed by a member node $j$ during session $i$ is computed by equation (4). 


\begin{tabular}{|c|c|c|c|c|c|c|}
\hline 2 octets & 1 octets & 1 octets & 1 octets & 1 octets & 1 octets & 2 octets \\
\hline \begin{tabular}{|l|} 
Frame \\
Control
\end{tabular} & \begin{tabular}{|l|} 
Node $_{1}{ }^{\prime} \mathrm{s}$ \\
Control \\
slot $\left(\mathrm{CS}_{1}\right)$
\end{tabular} & \begin{tabular}{|l|} 
Node $_{2}{ }^{2} \mathrm{~s}$ \\
Control \\
slot $\left(\mathrm{CS}_{2}\right)$
\end{tabular} & $\begin{array}{l}\text { Node }_{m}{ }^{\prime} \text { s } \\
\text { Control } \\
\text { slot }\left(\mathrm{CS}_{\mathrm{n}}\right)\end{array}$ & \begin{tabular}{|l} 
Control \\
Slot \\
duration \\
(CSD)
\end{tabular} & $\begin{array}{l}\text { Number } \\
\text { of slots }\end{array}$ & FCS \\
\hline
\end{tabular}

Fig. 2: Frame format of CS_Assign message.

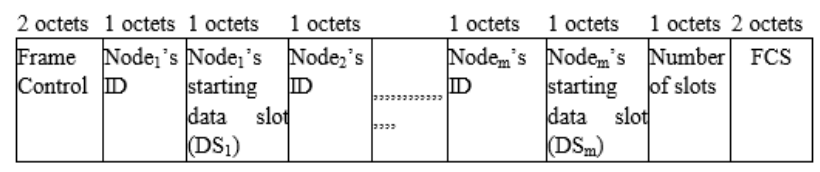

Fig. 3: Frame format of DS_Alloc message.

$$
E_{j}^{S S P_{i}}=(N-1) P_{j}^{C P_{i}} \times T_{C P}+P_{j}^{D S A_{i}} \times T_{D S A}+x_{j} \times P_{j}^{D S_{i}} \times T_{D S}
$$

where, parameters $P_{j}^{C P_{i}}, P_{j}^{D S A_{i}}$, and $P_{j}^{D S_{i}}$ indicate power consumed by member node $j$ in transmitting Data_Req message, receiving DS_Alloc message and transmitting data packets. The parameters $T_{C P}, T_{D S A}$, and $T_{D S}$ indicate the time required to transmit Data_Req message, receive DS_Alloc message and transmit data packets, and $x_{j}$ are the number of data slots allocated to member node $j$.

Energy consumed by $\mathrm{CH}$ in steady-state phase during session $i$ is calculated by equation (5).

$$
E_{c h}^{S S P_{i}}=(N-1) P_{c h}^{C P_{i}} \times T_{C P}+P_{c h}^{D S A_{i}} \times T_{D S A}+\sum_{j=1}^{N-1} x_{j} \times P_{c h}^{D S_{i}} \times T_{D S}
$$

where, $P_{c h}^{C P_{i}}, P_{c h}^{D S A_{i}}$, and $P_{c h}^{D S_{i}}$ are power consumed by cluster head in receiving Data_Req message, transmitting DS_Alloc message and receiving data packets. The $T_{C P}, T_{D S A}$, and $T_{D S}$ are the time utilized to receive Data_Req message in control slot, transmit DS_Alloc message and receive data packets respectively, and $x$ are the variable time slots assigned to the sensor nodes.

Each round consists of $l$ sessions, thus total energy consumption during SSP is computed by equation (6).

$$
E^{S S P}=\sum_{i=1}^{l}\left(\sum_{j=1}^{N-1} E_{j}^{S S P_{i}}+E_{c h}^{S S P_{i}}\right)
$$

\section{Simulation Results and Analysis}

In this section, we analyze the effectiveness of ATDMA-MAC protocol against the existing TDMA based MAC protocols in terms of data transmitted, energy efficiency and transmission delay. In the simulation, we consider one cluster which contains 10 sensor nodes and one $\mathrm{CH}$. Probability P indicated the number of nodes having data requests for packets transmission. In the steady-state phase, there are $\mathrm{k}$ sessions in each round, where $\mathrm{k}=2$. The data traffic is randomly generated by nodes within the range of 185 Bytes to $2.85 \mathrm{~KB}$. Each sensor is assumed to have an initial energy of 0.5 joules. The transmitting power is set to $426 \mathrm{~mW}$, receiving power is set to $346 \mathrm{~mW}$, and idle listening power is set to $330 \mathrm{~mW}$. The network is defined to have $250 \mathrm{kps}$ bandwidth with data slot length of $0.0083 \mathrm{sec}$, and control slot length of $0.00133 \mathrm{sec}$.

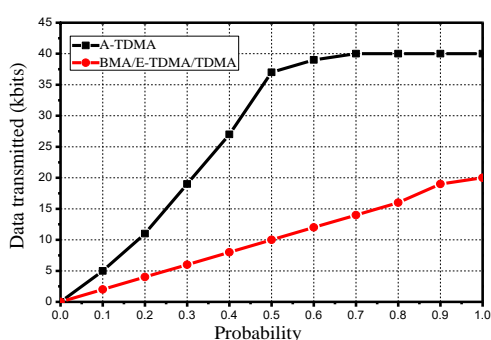

(a)

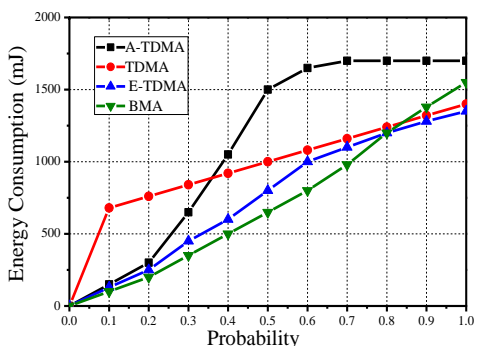

(b)

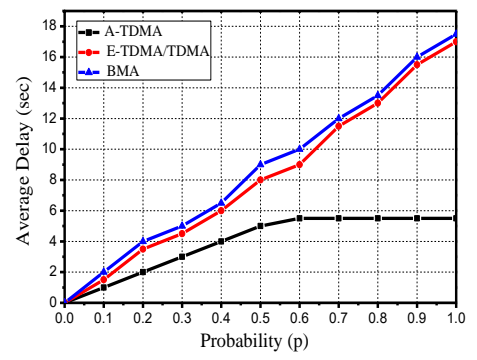

(c)

Fig. 4: Performance evaluation parameters with varying probability (a) Transmitted data (b) Energy consumption (c) Average Delay.

Fig. 4 (a) shows the amount of data transmitted by the member nodes for varying probability. It is noticed that ATDMA-MAC protocol transmits more data as compared to TDMA, E-TDMA, and BMA protocols when the probability of transmitted source nodes increases. This improvement in data transmission is due to the accumulation of variable traffic load in a large number of dynamic time slots based on their queue length information.

Fig. 4 (b) shows the total energy consumed by the $\mathrm{CH}$ and cluster member nodes versus probability. It is noticed that the energy consumption in ATDMA-MAC is minimized as compared to other TDMA based 
MAC protocols while transmitting the same amount of data. However, when the probability becomes 0.8 , the energy consumption of ATDMA-MAC remains constant, as it is not able to transmit more data for 2 sessions.

Fig. 4 (c) shows the transmission delay associated with the sensor nodes for varying probability. It is observed that ATDMA-MAC has less transmission delay as compared to BMA, E-TDMA, and TDMA protocols. This is due to the integration of adaptive TDMA scheduling in which all nodes send their data at once regardless of transmitting in small parts. As a consequence, the nodes keep data in their buffer for a smaller time. The results show that average latency of ATDMA-MAC is minimized up to $62 \%$ and $68 \%$, compared to BMA and E-TDMA, respectively.

\section{Conclusion}

In this paper, an adaptive TDMA scheduling based MAC protocol called ATDMA-MAC has been proposed to provide QoS assurance to delay sensitive data traffic in WSN. The ATDMA-MAC protocol efficiently handles the variable traffic load by dynamically assigning time slots to the sensor nodes based on their queue length information. The performance of the ATDMA-MAC protocol is compared with related TDMA based MAC protocols namely, TDMA, E-TDMA and BMA through simulations. The result shows that ATDMA-MAC protocol achieves high efficiency in data transmission delay along with minimum energy consumption.

\section{References}

[1] V. Bhandary, A. Malik, and S. Kumar. Routing in wireless multimedia sensor networks: a survey of existing protocols and open research issues. Journal of Engineering, 2016, pp. 1-28.

[2] J. Yick, B. Mukherjee, and D. Ghosal. Wireless sensor network survey. Computer networks. 2008, 52(12), pp.2292-2330.

[3] M.A. Yigitel, O.D. Incel, O.D. and C. Ersoy. QoS-aware MAC protocols for wireless sensor networks: A survey. Computer Networks. 2011, 55(8), pp.1982-2004.

[4] A. Sahoo and S. Chilukuri. DGRAM: a delay guaranteed routing and MAC protocol for wireless sensor networks. IEEE Transactions on Mobile Computing, 2010, 9(10), pp.1407-1423.

[5] P. Park, P. Di Marco, C. Fischione, and K.H. Johansson. Modeling and optimization of the IEEE 802.15. 4 protocol for reliable and timely communications. IEEE Transactions on Parallel and Distributed Systems. 2013, 24(3), pp.550-564.

[6] A. Bachir, M. Dohler, T. Watteyne, and K.K. Leung, 2010. MAC essentials for wireless sensor networks. IEEE Communications Surveys \& Tutorials. 2010, 12(2), pp.222-248.

[7] W. Ye, J. Heidemann, and D. Estrin, 2002. An energy-efficient MAC protocol for wireless sensor networks. In Proc. of 21 Ist IEEE Annual Joint Conference on Computer and Communications Societies. 3, pp. 1567-1576.

[8] T. Van Dam and K. Langendoen. An adaptive energy-efficient MAC protocol for wireless sensor networks. In Proc. of the 1st international conference on Embedded networked sensor systems. 2003, pp. 171-180.

[9] J. Polastre, J. Hill, and D. Culler. Versatile low power media access for wireless sensor networks. In Proc. of the 2nd international conference on Embedded networked sensor systems. 2004, pp. 95-107.

[10] W.R. Heinzelman, A. Chandrakasan, and H. Balakrishnan. Energy-efficient communication protocol for wireless microsensor networks. In Proc. of 33rd Hawaii international conference on System Sciences. 2000, pp. 10-16.

[11] T. Kaur and D. Kumar. TDMA-based MAC protocols for wireless sensor networks: A survey and comparative analysis. In Proc. of $5^{\text {th }}$ IEEE Confernce on Wireless Networks and Embedded Systems (WECON). 2016, (pp. 1-6).

[12] W.B. Heinzelman, A.P. Chandrakasan, and H. Balakrishnan. An application-specific protocol architecture for wireless microsensor networks. IEEE Transactions on wireless communications. 2000, 1(4), pp.660-670.

[13] J. Li, and G.Y. Lazarou. A bit-map-assisted energy-efficient MAC scheme for wireless sensor networks. In Proc. of the 3rd international symposium on Information processing in sensor networks, 2004, pp. 55-60.

[14] T.H. Hsu and P.Y. Yen. Adaptive time division multiple access-based medium access control protocol for energy conserving and data transmission in wireless sensor networks. IET communications. 2011, 5(18), pp.2662-2672. 